July I 2014

IPS Welsh Branch National Conference

Mercure Holland House Hotel, Cardiff

Website: www.ips.uk.net

July 22014

IPS East of England Branch Conference

Kings Gate Conference Centre, Peterborough

Website: www.ips.uk.net

\section{July 92014}

IPS West Midlands Branch Conference

Bethel Convention Centre, Birmingham

Website: www.ips.uk.net

September 29 - October 12014

Infection Prevention 2014

SECC, Glasgow

Website: www.ips.uk.net

\section{October 12014}

IPS Carehome Conference

SECC, Glasgow

Website: www.ips.uk.net
October I - 32014

International Conference on Antimicrobial Research

Madrid

Website: http://www.icar-2014.org/

\section{October 20-22 2014}

Vaccines 2014

Cineworld: The O2, Peninsula Square, London, SE 10 ODX

Website: enquiries@euroscicon.com

November 16 - 182014

\section{HIS 2014}

Lyon Convention Centre, Lyon, France

Website: http://www.his.org.uk/events/his2014/

\section{November 252014}

4th Annual IV Forum Conference

Kings Gate Conference Centre, Peterborough

Website: www.ips.uk.net
To place an event in this free listings section email the details of your event to: pa@ips.uk.net 